\title{
Experimental and Numerical Studies of Paraguayan Chaco Natural Gas in a Counterflow Diffusion Flame
}

\author{
Hernando Maldonado, Dario Alviso, Miguel Mendieta, Juan Carlos Rolón \\ Facultad de Ingeniería, Universidad Nacional de Asunción, San Lorenzo, 2160 Paraguay.
}

Received: May 13, 2015 / Accepted: June 10, 2015 / Published: September 25, 2015.

\begin{abstract}
Combustion is a chemical phenomenon in which a multitude of elementary chemical reactions take place, resulting in the overall process of fuel oxidation. Natural gas fuel has been explored for a few decades and extracted for a few years in the region of Paraguayan Chaco, near Bolivia border. Currently, natural gas is not very important in Paraguay's energy matrix, however it could be in the near future if higher volumes are extracted and transported to the most populated cities, specially to the capital. In order to improve Paraguayan natural gas combustion performance, an understanding of its fundamental properties and the combustion pathways is required. This study presents new data for Paraguayan Chaco natural gas combustion in a laminar counterflow diffusion flame configuration at atmospheric pressure. Visible chemiluminescence of excited radicals $\mathrm{CH}^{*}$ and $C_{2}^{*}$ is employed experimentally. 1D numerical simulation was carried out using Paraguayan Chaco natural gas chemical composition and a standard kinetic mechanism, to which we added $\mathrm{CH}^{*}$ and $\mathrm{C}_{2}^{*}$ reactions. Typical flame structures resulting from simulation are presented and a validation of the model is realized comparing experimental and numerical $\mathrm{CH}^{*}$ and $\mathrm{C}_{2}^{*}$ radicals profiles.
\end{abstract}

Key words: Natural gas, counterflow, diffusion flames, numerical simulation, kinetic modeling, chemiluminescence

\section{Introduction}

Mastering energy is a key factor for human to preserve its economic growth and maintain his life standard. According to the International Energy Agency (IEA) report and Shahid et al. [1], the world will increase by $40 \%$ its energy requirements in 2035.

Taking into account all primary energy produced around the world, combustion of liquid, gas and solid fuels corresponds to about $85 \%$ of the total. These resources are non-renewable, therefore combustion efficiency is one of the way to overcome this limitation. In the same time, due to climate changes, lower emissions to the atmosphere are one of the main requirements nowadays in most combustion systems and a challenge for research.

This work aims to study the combustion of

Corresponding author: Hernando Arturo Maldonado Colmán, Engineer, research field: combustion. E-mail: hmaldonado@ing.una.py.
Paraguayan Chaco natural gas, from experimental and numerical point of view, for which the counterflow diffusion (non-premixed) flame configuration at atmospheric pressure and constant injection temperature was chosen.

Combustion is a chemical phenomenon in which a multitude of elementary chemical reactions take place, resulting in the overall process of fuel oxidation and reduction. During these elementary chemical reactions, the chemical species are transformed into other species. These reaction intermediates may absorb part of the reaction energy placing them in an excited state. A possible way of de-excitation is the spontaneous emission of photons from the populated state of the specific species, called chemiluminescence. Chemiluminescence is the emission of light by molecules returning to their ground state after being excited chemically, rather than thermally. The emission shows a weak dependence on temperature and a greater dependence on chemical composition. One way to understand these complex chemical 
processes is to follow the evolution of chemiluminescence of specific species produced in the reaction processes.

In order to acquire a better understanding of combustion fundamental phenomena, it is preferable to study flames configurations with flows relatively simple that are easy to control.

The counterflow laminar flames meet these criteria: the flow is well known, it can be modeled from a theoretical point of view and experimentally it can be controlled in a relatively easy manner.

The interest of these stretched flames also lies in the fact that they act as the main element to describe some turbulent combustion models.

The primary combustion zone of hydrocarbon flames is distinct from the other parts of the flame by its emission of mostly visible bands, namely $\mathrm{CH}$ bands at $431 \mathrm{~nm}\left(A^{2} \boldsymbol{\Delta} \rightarrow X^{2} \Pi\right)$ and $C_{2}$ band at $516 \mathrm{~nm}\left(d^{3} \Pi_{g} \rightarrow a^{3} \Pi_{u}\right)$ (Swan band).

A convenient way to experimentally study the flame behavior is to analyze the space and time resolved emission of excited species $\mathrm{CH}^{*}$ and $\mathrm{C}_{2}^{*}$, which are not at equilibrium under flame conditions. Indeed, these radicals are naturally present in the combustion zone and permit to determine important macroscopic properties such as flame location, flame speed, equivalence ratio and heat release rate fluctuations.

We intend to study two flames operating conditions (equivalence ratio and strain rate), for which the existing experimental and computational equipments of the Mechanical and Energy Laboratory were used. The flame structure of natural gas diffusion flame will be analyzed. $\mathrm{CH}^{*}$ and $\mathrm{C}_{2}^{*}$ experimental and numerical profiles will be compared to validate the chosen chemical mechanism as well as added $\mathrm{CH}^{*}$ and $C_{2}^{*}$ reactions.

\section{Experimental Configuration}

\subsection{Natural Gas Chemical Composition}

The natural gas chemical composition was analyzed using Gas Chromatography and Mass Spectrometry (GC-MS) techniques. This information is needed to estimate the equivalence ratio of natural gas flames, and also is used as input values to carry out numerical simulations.

The estimation of natural gas composition is presented in Table 1. In comparison to literature values, it corresponds to the classical composition of this fuel, where the major component is methane, with some minor components, as shown in Table 1. 'Others' (with a mole fraction $\mathrm{x} 100$ of 0.987 ) refers to species not present in the chosen kinetic scheme. This point will be discussed in Section 3.2.

\subsection{Counterflow Burner Device}

Flames experiments were carried out in a counterflow burner. This configuration is briefly described in the next paragraph. More information of the burner can be found in $[2,3]$.

The burner consists of two opposed axisymmetric convergent nozzles of $20 \mathrm{~mm}$ inner diameter (see Figure 1A). A flow of air is injected at ambient temperature in the upper side of the burner, while a mixture of natural gas and nitrogen is transported from the lower side, also at ambient temperature. Nitrogen component in this mixture is added in order to compensate the momentum of the air flowing from the upper side of the burner. After ignition, a laminar diffusion flame is formed between the nozzles. The distance between the two nozzle exits was kept constant to $20 \mathrm{~mm}$. Each nozzle contains two layers of honeycomb to reduce turbulent structures in the plenum chamber of the nozzle. Each nozzle is

Table 1 Composition of Paraguayan Chaco natural gas.

\begin{tabular}{ll}
\hline \multicolumn{1}{c}{ Paraguayan Chaco Gas Composition } \\
\hline Components & Mole fraction x 100 \\
\hline Nitrogen & 3.905 \\
Carbon Dioxide & 0.030 \\
Methane & 94.277 \\
Ethane & 1.611 \\
Others & 0.987 \\
Total & 100 \\
\hline
\end{tabular}


surrounded by a coaxial nozzle fed by nitrogen, which isolates the reaction zone from ambient perturbations that could disturb the measurements.

In order to limit the thermal effect of the burnt gases circulating in the upper zone of the system, the upper burner has a thermal screen, as well as an isolator (glass wool) with plaster to optimize the temperature uniformity, as one can see in Figure 1A.

Two natural gas flames operating conditions have been chosen, considering the equivalence ratio and the strain rate: Flame I, with an equivalence ratio $\phi=4$ and strain rate $\varepsilon=134 \mathrm{~s}^{-1}$, and Flame II, with an equivalence ratio $\phi=3.5$ and strain rate $\varepsilon=$ $136 s^{-1}$. The injection temperature was kept constant throughout the study in both nozzle exits (Injection Temperature $=300 \mathrm{~K})$.

Strain rates $(d V / d z)$ are estimated using the following equation:

$$
\varepsilon=\frac{2 V_{u}}{H}\left(1+\frac{V_{l}}{V_{u}} \sqrt{\frac{\rho_{l}}{\rho_{u}}}\right)
$$

Where $V_{u}, V_{l}, \rho_{u}, \rho_{l}$ are the upper and lower nozzle exits velocities and densities, respectively. $H$ is the distance between the two nozzle exits.

All gaseous mass flow rates are controlled by the mass flow meters with an accuracy of $\pm 1.5 \%$ and a repeatability of $\pm 0.5 \%$ of the full scale. The overall uncertainty of strain rates and natural gas flames equivalence ratios can therefore be estimated at $\pm 0.78 \%$ and $\pm 1.52 \%$, respectively.

\subsection{Visualization}

Images of $\mathrm{CH}^{*}$ and $\mathrm{C}_{2}^{*}$ emission have been recorded using a $576 \times 384$ pixels ICCD camera (Princeton Instruments) equipped with a lens (Nikkor, $105 \mathrm{~mm}$ focal length) (see Figure 1B).

Narrow-band interference filters were interposed along the optical path for capturing $\mathrm{CH}^{*}$ and $\mathrm{C}_{2}^{*}$ emission. The filter used for $\mathrm{CH}^{*}$ has $60 \%$ transmission and a $10 \mathrm{~nm}$ wide bandpass centered around $430 \mathrm{~nm}$. The $C_{2}^{*}$ filter is centered at $515 \mathrm{~nm}, 30 \mathrm{~nm}$ bandpass and $70 \%$ transmission in

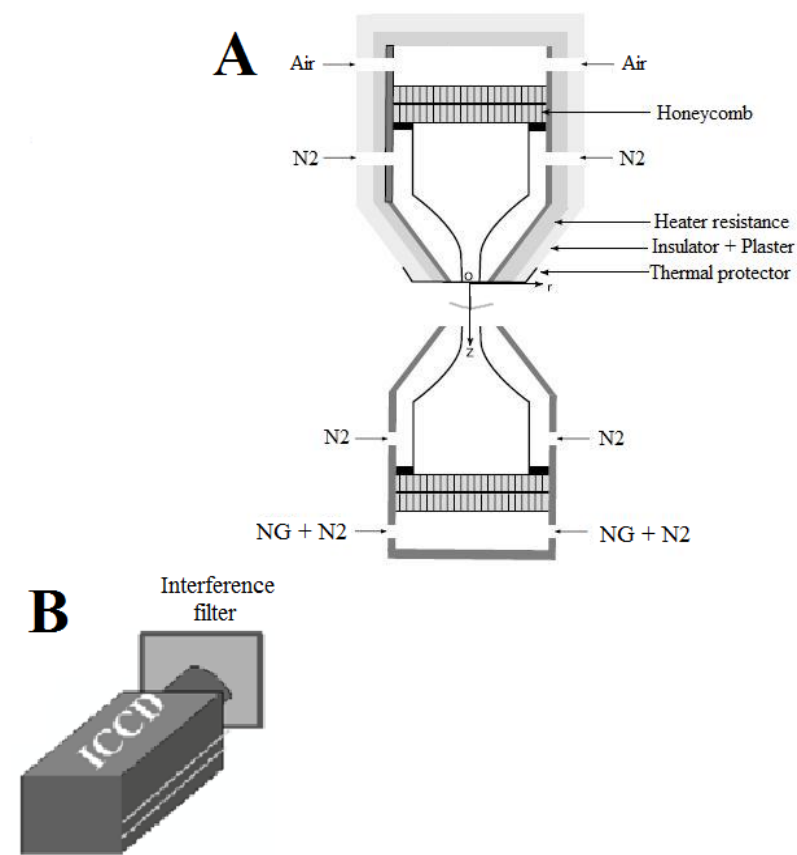

Fig. 1 (A) Counterflow burner device, (B) Visualization setup.

the maximum. The exposition time was kept constant at $30 \mathrm{~ms}$.

Figure 2A presents a typical natural gas flame front obtained from $\mathrm{CH}^{*}$ visualization. This figure shows that the flame front is slightly convex due to gravity effects, but near the normal axial direction to the flame surface it remains plane and similarity hypothesis may be applied.

The line of sight creates an integrated signal along its trajectory and increases the emitted signals. As the flame front is axis-symmetrical, Abel integral inversion is used to eliminate the effects of the integration and get the tomography trace of the flame front in the symmetry plane of the burner as done in $[4$, 5] (Fig. 2B, Fig. 3).

Axial profiles of both $C^{*}$ and $C_{2}^{*}$ are normalized to corresponding maximum peaks. Due to experimental difficulties, emission intensity calibration was not performed. Therefore, the comparison with numerical profiles will be done considering relative intensities of the position of both $\mathrm{CH}^{*}$ and $\mathrm{C}_{2}^{*}$ radicals, and not the absolute concentration. 


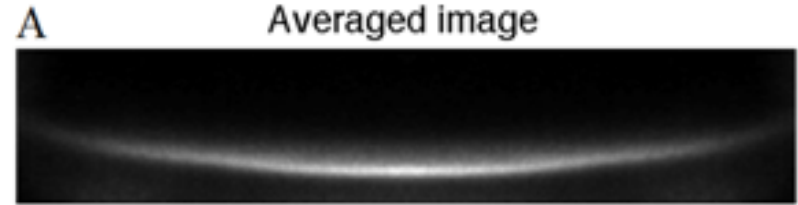

\section{B Abel Inversion of the averaged image}

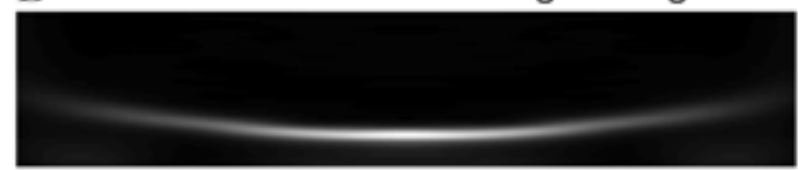

Fig. 2 (A) Averaged image of Flame I $\mathrm{CH}^{*}$ emission, (B) Abel Inversion of the averaged image of Flame I $\mathrm{CH}^{*}$ emission.

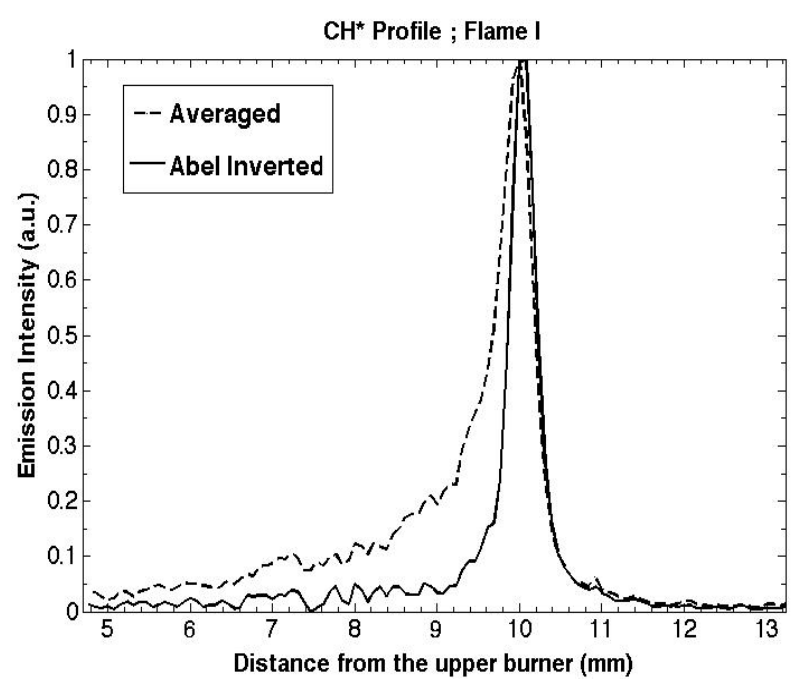

Fig. 3 Averaged (Integrated in the line of sight) and Abel inverted axial profiles of Flame I $\mathrm{CH}^{*}$ emission.

The resolution of the CCD camera along the flame axis is $77 \mu \mathrm{m}$. Then, due to the slight flames oscillation, the flame position was estimated with an accuracy of $\pm 115 \mu \mathrm{m}$.

\section{Numerical Simulation}

\subsection{Governing Equations}

We consider an axisymmetric counterflow configuration shown in Figure 4. Air is injected from the left side whereas a mixture of natural gas and nitrogen is injected from the right side. We model our system using similarity approach by searching for similar solutions of gaseous flow equations in the vicinity of the central axis [7]. These similar solutions have the form: gas density $\rho=\rho(z)$, gas radial velocity $u=r U(z)$, gas axial velocity $v=v(z)$, gas temperature $T=T(z)$ and species mass fractions $Y_{k}=Y_{k}(z), k=1, \ldots N_{s p} \quad\left(N_{s p}\right.$ is the number of species).

By assuming a pressure gradient in the radial direction so that $-\frac{1}{r} \frac{\partial p}{\partial z}=J$ is constant along the $z$ axis, the gaseous phase are described by the following balance equations [7]:

$$
\begin{gathered}
2 \rho U+\frac{\partial \rho v}{\partial z}=0 \\
\rho U^{2}+\rho v \frac{\partial U}{\partial z}=\frac{\partial}{\partial z}\left(\mu \frac{\partial U}{\partial z}\right)+J \\
\rho v c_{p} \frac{\partial T}{\partial z}=\frac{\partial}{\partial z}\left(\lambda \frac{\partial T}{\partial z}\right)- \\
\sum_{k=1}^{K} h_{k} W_{k} \Omega_{k}-\left(\sum_{k=1}^{K} Y_{k} V_{k z} c_{p k}\right) \frac{\partial T}{\partial z} \\
\rho v \frac{\partial Y_{k}}{\partial z}=-\frac{\partial}{\partial z}\left(\rho Y_{k} V_{k z}\right)+W_{k} \Omega_{k}, \\
k=1, \ldots, N_{s p}
\end{gathered}
$$

In these equations $\mu$ is the gaseous mixture viscosity. $c_{p k}$ and $c_{p}$ are the heat capacity at local constant pressure of species $k$ and of the mixture respectively. $h_{k}, W_{k}, \Omega_{k}$ are the specific enthalpy, the molar weight and the molar chemical production rate of the $k^{t h}$ species respectively, and $V_{k z}$ is the diffusion velocity of the $k^{\text {th }}$ species in the axial direction.

The above system of equations is completed by the ideal gas equation and by specifying that $\frac{\partial J}{\partial z}=0$.

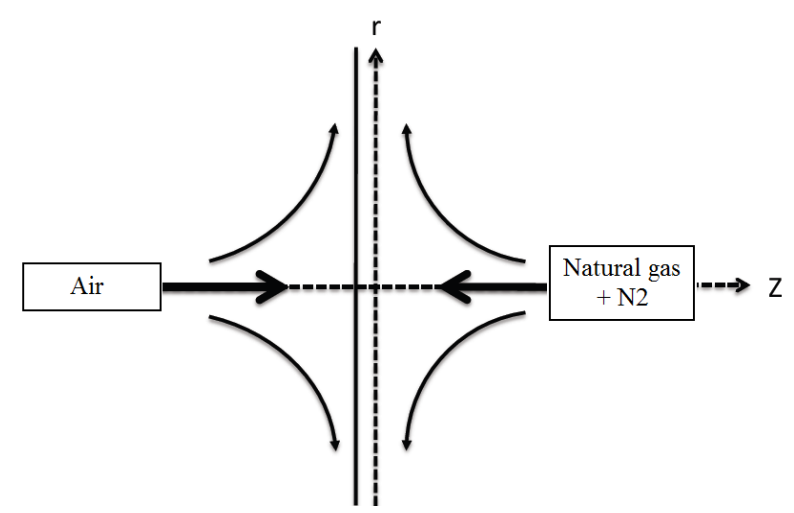

Fig. 4 Counterflow diffusion flame configuration. 


\subsection{Numerical Conditions}

The boundary conditions of the system are given in Table 2.

The set of equations is then replaced by a fully coupled set of discrete relations. The solution of this system is base on a global adaptive nonlinear method using Newton iterations (see [8,9]). The grid is adapted to first and second order derivatives of all variables and the smallest grid size $5 \mu \mathrm{m}$.

The kinetic modeling for natural gas oxidation in the counterflow flame was performed using the REGATH-1D-COUNTERFLOW code of the REGATH package with detailed thermochemical and transport properties [7]. The inputs to each simulation include a chemical kinetic reaction mechanism, a dataset of thermochemical properties and a dataset of transport properties.

A methane mechanism (Lindstedt [10] including 29 species and 141 reactions) was chosen to carry the simulations. We have chosen this mechanism for the natural gas/air combustion due to the composition of this fuel (see Table 1), where it can be seen that methane is the main component of this fuel. In order to take into account natural gas composition, species $\mathrm{N}_{2}, \mathrm{CO}_{2}, \mathrm{CH}_{4}$ and $\mathrm{C}_{2} \mathrm{H}_{6}$ were introduced as input. Furthermore, the mole fractions of these species were normalized in order to have the total mole fraction equals 1 ('Others' species are not present in Lindstedt chemical scheme).

\section{$3.3 \mathrm{CH}^{*}$ and $\mathrm{C}_{2}^{*}$ Reactions}

$\mathrm{CH}^{*}$ and $\mathrm{C}_{2}^{*}$ species are not present in Lindstedt [10] mechanism, therefore chemical reactions describing the production, quenching and chemiluminescence of $\mathrm{CH}^{*}$ and $\mathrm{C}_{2}^{*}$ were added to this mechanism. Also species $C_{3}$ was introduced since it appears in $\mathrm{C}_{2}^{*}$ production reactions.

As expected, due to the low concentration of the excited species, no significant difference in the results was found with the addition of these elementary reactions. The thermochemical data for $\mathrm{CH}^{*}$ and $\mathrm{C}_{2}^{*}$
Table 2 Boundary conditions.

\begin{tabular}{lcc}
\hline & Left $(z=-10)$ & Right $(z=10)$ \\
\hline Gas Temperature & $T_{g}=T_{g}^{\text {left }}$ & $T_{g}=T_{g}^{\text {right }}$ \\
Species mass fractions & $Y_{k}=Y_{k}^{\text {left }}$ & $Y_{k}=Y_{k}^{\text {right }}$ \\
Gas axial velocity & $v_{g}=v_{g}^{\text {left }}$ & $v_{g}=v_{g}^{\text {right }}$ \\
Gas radial velocity & $u_{g}=0$ & $u_{g}=0$ \\
\hline
\end{tabular}

were added as well. The transport coefficients for the excited species were the same as those of the ground state species.

For species $\mathrm{CH}^{*}$ and specially for $\mathrm{C}_{2}^{*}$ literature data is very scarce. However, Kathrotia et al. [11] recently presented production and destruction reactions for these species. Therefore, [11] was chosen for estimating $\mathrm{CH}^{*}$ and $\mathrm{C}_{2}^{*}$ species concentration.

\section{Results and Discussion}

\subsection{Natural Gas Flame Structure}

Figure 5 presents a typical natural gas flame structure. The boundary conditions correspond to the Flame I (see Section 2.2). In this figure $z=-10$ corresponds to the upper burner edge and $z=10$ to the lower burner edge. The diffusion natural gas/air flame front is located at about $z=0.1 \mathrm{~mm}$. At this point, the gas temperature passes through a maximum $(1820 \mathrm{~K})$.

In Figure 5 (top), methane $\left(\mathrm{CH}_{4}\right)$ species profile remains constant until $z=3 \mathrm{~mm}$, then decreases rapidly before reaching the natural gas/air flame front. On the left side of the figure, Oxygen $\left(\mathrm{O}_{2}\right)$ species profile remains constant until $z=-2 \mathrm{~mm}$ and then also decreases rapidly before reaching the natural gas/air flame front. In this type of flame, molecular diffusion between species is the most important phenomenon.

In Figure 5 (bottom), water vapor $\left(\mathrm{H}_{2} \mathrm{O}\right)$ species profile is presented, and as it can be seen the profile is very similar to that of temperature. $\mathrm{H}_{2} \mathrm{O}$ is one of the main products of natural gas combustion. In hydrocarbons flames reactions usually begin with hydrogen $(H)$ abstraction. In natural gas, methane $\left(\mathrm{CH}_{4}\right)$ is the main component; therefore hydrogen $(\mathrm{H})$ 

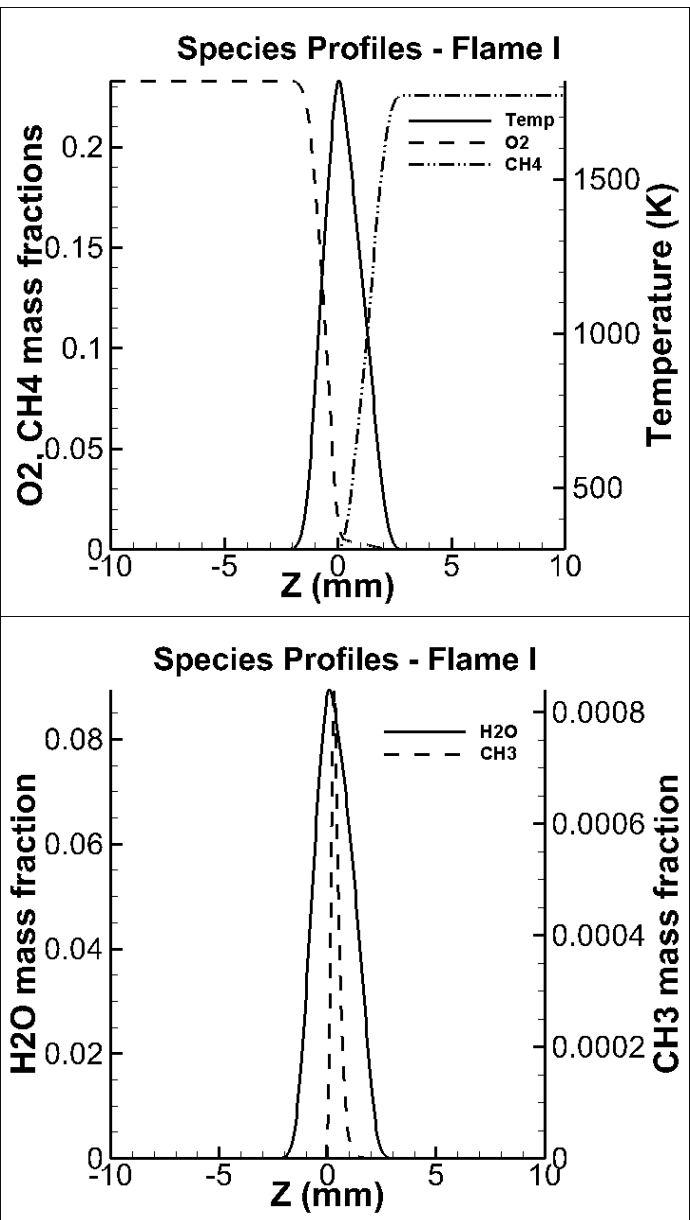

Fig. 5 Species and temperature profiles of natural gas, Flame I $\left(\phi=4\right.$ and $\left.\varepsilon=134 s^{-1}\right)$.

abstraction produces methyl radical $\left(\mathrm{CH}_{3}\right)$. Consequently, as $\mathrm{CH}_{4}$ starts to decrease at about $z=3 \mathrm{~mm}$ (Figure 5 (bottom)), $\mathrm{CH}_{3}$ starts to increase. Methyl radical in turn decomposes into other radicals, explaining its presence in a thin region.

\subsection{Experimental and Numerical $\mathrm{CH}^{*}$ and $\mathrm{C}_{2}^{*}$ Axial Profiles}

Figure 6 gives a comparison between experimental and numerical profiles of $\mathrm{CH}^{*}$ (top) and $\mathrm{C}_{2}^{*}$ (bottom) radicals for the natural gas/air Flame I.

As the measurements do not give absolute values, in order to make comparisons with the numerical results, the experimental profiles of $\mathrm{CH}^{*}$ and $\mathrm{C}_{2}^{*}$ were normalized by their maximum values. In Figure 7 is presented the same comparison but with the profiles of Flame II.
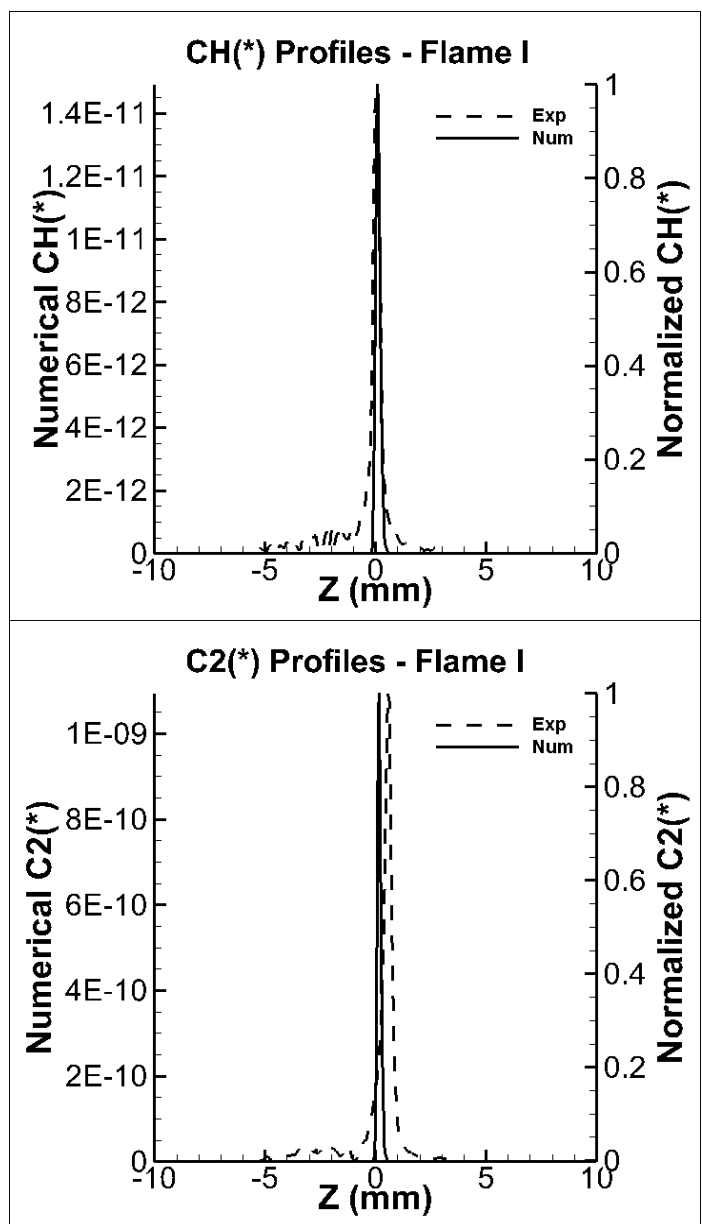

Fig. 6 Experimental and numerical $C^{*}$ (top) and $C_{2}^{*}$ (bottom) species profiles, Flame $I$ ( $\phi=4$ and $\varepsilon=$ $\left.134 s^{-1}\right)$.

As we can see in Figures 6 and 7 (top), the numerical predictions of $\mathrm{CH}^{*}$ are very similar to the experimental profiles along the axis. The positions of the peaks match almost perfectly. The experimental profiles are slightly thicker than the simulated ones, however this can be explained by the poorer spatial resolution in the experiments $(77 \mu \mathrm{m})$.

However, as we can see in Figures 6 and 7 (bottom), the numerical predictions of $C_{2}^{*}$ are slightly displaced compared to those of the experimental profiles along the axis. For Flame I, the difference between the numerical and experimental $C_{2}^{*}$ is less than $1 \mathrm{~mm}$.

For Flame II, the difference is slightly higher than $1 \mathrm{~mm}$. Considering the experimental errors presented in Sections 2.2 and 2.3 and taking into account the distance between the two burners $(20 \mathrm{~mm})$, these 

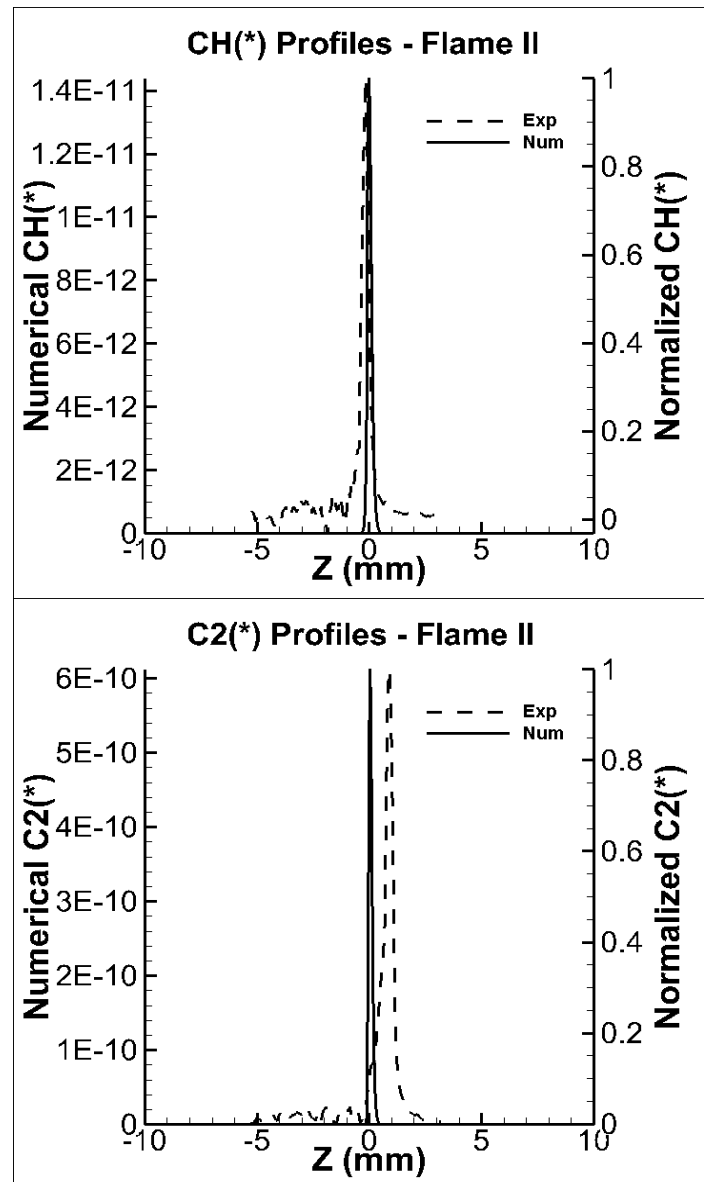

Fig. 7 Experimental and numerical $\mathrm{CH}^{*}$ (top) and $C_{2}^{*}$ (bottom) species profiles, Flame II $(\phi=3.5$ and $\left.\varepsilon=136 s^{-1}\right)$

differences are not very important, therefore this good agreement between experimental and numerical $\mathrm{CH}^{*}$ and $C_{2}^{*}$ radicals validates the chosen kinetic mechanism, and consequently the reactions of these radicals added to it.

\section{Conclusions}

Paraguayan Chaco natural gas combustion in a laminar counterflow diffusion flame configuration at atmospheric pressure was studied.

The visible chemiluminescence of the excited radicals $\mathrm{CH}^{*}$ and $C_{2}^{*}$ is employed experimentally.

Numerically, a 1D simulation was carried out using a standard kinetic mechanism, to which we add $\mathrm{CH}^{*}$ and $C_{2}^{*}$ reactions.

Typical natural gas flame structure was presented.

A comparison between experimental and numerical profiles of $\mathrm{CH}^{*}$ and $C_{2}^{*}$ radicals for the natural gas/air was done for two operating conditions, validating the model chosen in this study, and the reactions of these excited radicals added to it.

\section{References}

[1] E. M. Shahid, Y. Jamal. Production of biodiesel: A technical review. Renewable and Sustainable Energy Reviews (2007). Vol 15. 9:4732-4745.

[2] J. C. Rolon, D. Veynante, J. P. Martin, F. Durst. Counter jet stagnation flows. Experiment in Fluids (1991). Vol 11. 313-324.

[3] J. C. Rolon, F. Aguerre, S. Candel. Experiments on the Interaction between a Vortex and a Strained Diffusion Flame. Combustion and Flames (1995). Vol 100. 422-429.

[4] M. De Leo, A. Saveliev, L. A. Kennedy, S. A. Zelepouga. $\mathrm{OH}$ and $\mathrm{CH}$ luminescence in opposed flow methane oxy-flames. Combustion and Flames (2007). Vol 149. 435-447.

[5] G. Herding, R. Snyder, J. C. Rolon, S. Candel. Investigation of cryogenic propellant flames using computerized tomography of $\mathrm{OH}$ emission images. Journal of Propulsion and Power (1998). Vol 13. 146-151.

[6] D. Alviso, J. C. Rolon, P. Scouflaire, N. Darabiha. Experimental and numerical studies of biodiesel combustion mechanisms using a laminar counterflow spray premixed flame. Fuel, Volume 153, 2015, Pages 154-165

[7] N. Darabiha, F. Lacas, J. C. Rolon, S. Candel. Laminar counterflow spray diffusion flames: A comparison between experimental results and complex chemistry calculations. Combustion and Flame (1993). Vol 95. 261-275.

[8] N. Darabiha, S. Candel, V. Giovangigli, M. D. Smooke. Extinction of Strained Premixed Propane-air Flames with Complex Chemistry. Combustion Science and Technology (1988). Vol 60. 267-285.

[9] V. Giovangigli, M. D. Smooke. Extinction of Strained Premixed Laminar Flames with Complex Chemistry. Combustion Science and Technology (1987). Vol 53. 23-49.

[10] P. Lindstedt. Modeling of the chemical complexities of flames. Symposium (International) on Combustion (1998). Vol 27. 269-285.

[11] T. Kathrotia. Reaction Kinetics Modeling of $\mathrm{OH}^{*}, \mathrm{CH}^{*}$ and $\mathrm{C} 2 *$ Chemical luminescence. INAUGURALDISSERTATION zur Erlangung der Doktorwurde der Naturwisschenschaftlich-Mathematischen Gesamtfakultat der Ruprecht-Karls-Universitat Heidelberg (2011). 